\title{
Article \\ Impact of UGT1A1 Polymorphisms on Febrile Neutropenia in Pancreatic Cancer Patients Receiving FOLFIRINOX: A Single-Center Cohort Study
}

Jiyoung Keum ${ }^{1,2}$, Hee Seung Lee ${ }^{1}{ }^{\mathbb{D}}$, Jung Hyun Jo ${ }^{1}{ }^{(D}$, Moon Jae Chung ${ }^{1}$, Jeong Youp Park ${ }^{1}$, Seung Woo Park ${ }^{1}$, Si Young Song ${ }^{1}$ and Seungmin Bang ${ }^{1, *}$

1 Division of Gastroenterology, Department of Internal Medicine, Institute of Gastroenterology, Yonsei University College of Medicine, Seoul 03722, Korea; 01212@eumc.ac.kr (J.K.); lhs6865@yuhs.ac (H.S.L.); junghyunjo83@yuhs.ac (J.H.J.); mjchung@yuhs.ac (M.J.C.); sensass@yuhs.ac (J.Y.P.); swoopark@yuhs.ac (S.W.P.); sysong@yuhs.ac (S.Y.S.)

2 Division of Gastroenterology, Department of Internal Medicine, Ewha Womans University College of Medicine, Seoul 07985, Korea

* Correspondence: bang7028@yuhs.ac; Tel.: +82-2-2228-1995

check for updates

Citation: Keum, J.; Lee, H.S.; Jo, J.H.; Chung, M.J.; Park, J.Y.; Park, S.W.; Song, S.Y.; Bang, S. Impact of UGT1A1 Polymorphisms on Febrile Neutropenia in Pancreatic Cancer Patients Receiving FOLFIRINOX: A Single-Center Cohort Study. Cancers 2022, 14, 1244. https://doi.org/ 10.3390 /cancers 14051244

Academic Editor: Irene Esposito

Received: 11 February 2022 Accepted: 25 February 2022 Published: 28 February 2022

Publisher's Note: MDPI stays neutral with regard to jurisdictional claims in published maps and institutional affiliations.

Copyright: (C) 2022 by the authors. Licensee MDPI, Basel, Switzerland. This article is an open access article distributed under the terms and conditions of the Creative Commons Attribution (CC BY) license (https:// creativecommons.org/licenses/by/ $4.0 /)$.
Simple Summary: FOLFIRINOX, which is a first-line chemotherapy for metastatic pancreatic cancer, has become one of the high-risk regimens related to developing febrile neutropenia (FN). Although UGT1A1 polymorphisms are associated with the metabolism of irinotecan, their role as surrogate markers for FOLFIRINOX-induced neutropenia has not been confirmed. In this retrospective study, a total of 154 patients (FN group $(n=31)$ vs. non-FN group $(n=123)$ ) were divided into three groups based on the predicted UGT1A1 phenotype (extensive metabolizer (EM) vs. intermediate metabolizer (IM) vs. poor metabolizer (PM)). The Cox regression analysis showed that female sex (hazard ratio (HR): 2.20; $p=0.031)$, ECOG PS = 1 (HR: 2.83; $p=0.008)$, UGT1A1 IM (HR: 4.30; $p=0.004)$, and UGT1A1 PM (HR: 4.03; $p=0.028$ ) were independent risk factor of FN. We propose UGT1A1 as the strongest predictive factor for FN and the need for UGT1A1 screening prior to chemotherapy.

\begin{abstract}
FOLFIRINOX (oxaliplatin, leucovorin, irinotecan, and 5-fluorouracil) is a first-line chemotherapy for metastatic pancreatic cancer (PC). Chemotherapy-induced neutropenia is one of the most serious adverse events associated with advanced PC. Although UGT1A1 polymorphisms are associated with the metabolism of irinotecan, their role as surrogate markers for FOLFIRINOX-induced neutropenia has not been confirmed. We investigated risk factors for FN-in particular, UGT1A1 polymorphisms-in PC patients receiving FOLFIRINOX, using a single-center cohort registry. To investigate the association between UGT1A1 polymorphisms and FN, we divided patients into three groups based on the predicted UGT1A1 phenotype: extensive metabolizer (EM) vs. intermediate metabolizer (IM) vs. poor metabolizer (PM). A total of 154 patients (FN group $(n=31)$ vs. non-FN group $(n=123)$ ) receiving first-line FOLFIRINOX were identified between December 2017 and July 2020. The Cox regression analysis showed that female sex (HR: $2.20 ; p=0.031$ ), Eastern Cooperative Oncology Group performance status = 1 (HR: 2.83; $p=0.008)$, UGT1A1 IM (HR: 4.30; $p=0.004)$, and UGT1A1 PM (HR: 4.03; $p=0.028$ ) were statistically significant risk factors for FN. We propose that UGT1A1 is the strongest predictive factor for FN and that this gene should be screened prior to the administration of chemotherapy.
\end{abstract}

Keywords: pancreatic cancer; FOLFIRINOX; UGT1A1; febrile neutropenia; neutropenia

\section{Introduction}

Pancreatic cancer (PC) is a lethal malignant neoplasm, and surgical resection is the only curative treatment. Unfortunately, less than $20 \%$ of newly diagnosed PC patients are 
appropriate candidates for surgical resection [1]. Most other PC patients are diagnosed at an advanced stage and receive chemotherapy.

The FOLFIRINOX regimen, which consists of oxaliplatin, leucovorin, irinotecan, and 5-fluorouracil (5-FU), showed remarkably improved overall survival and objective tumor response rates in the PRODIGE4/ACCORD11 trial [2]. FOLFIRINOX has since become a first-line chemotherapeutic for metastatic PC [3,4].

However, the FOLFIRINOX regimen has unresolved problems regarding dose modification of chemotherapeutic agents and the use of prophylactic granulocyte colonystimulating factor (G-CSF) in relation to hematological adverse events (AEs), such as febrile neutropenia (FN) and severe neutropenia (NP). Previous studies reported that grade $3 / 4 \mathrm{NP}$ and FN developed in $45.7-77.8 \%$ and $5.4-22.2 \%$ of patients, respectively $[2,5,6]$. In particular, hematological AEs were more commonly seen in the Asian population than in the Western population $[5,7,8]$. In clinical practice, such a high probability of AEs is a reason for concern when using FOLFIRINOX. Based on the National Comprehensive Cancer Network (NCCN) guidelines version 4.2021, FOLFIRINOX has become one of the high-risk regimens related to the development of FN [9]. In this revised guideline, G-CSF is not routinely recommended as a primary prophylaxis but can be considered in patients with high-risk clinical characteristics.

There have been few studies on the high-risk population for FN occurrence in patients with PC receiving FOLFIRINOX. Two retrospective studies have reported female sex, overweight, initial biliary stent insertion, platelet count $\leq 15 \times 10^{4} / \mathrm{L}$, and heterozygosity for either the UGT1A1 ${ }^{*} 28$ or UGT1A1* 6 alleles as risk factors for FN in PC patients receiving FOLFIRINOX $[10,11]$. Irinotecan, one of the principal drugs of the FOLFIRINOX regimen, is a topoisomerase I inhibitor widely used in the treatment of cancer [12,13]. However, irinotecan presents a high incidence of toxicity, particularly severe NP, and diarrhea [14-16]. Irinotecan is converted to an active metabolite known as SN-38, subsequently inactivated and detoxified to an inactive form, $\mathrm{SN}-38$ glucuronide (SN-38G), by a uridine diphosphateglucuronosyl-transferase (UGT) enzyme encoded by the UGT1A1 gene [17]. The risk of irinotecan toxicity increases with the presence of genetic variants related to decreased UGT enzyme activity, including UGT1A1*28 and UGT1A1*6 [18-20]. These variants result in decreased excretion of irinotecan metabolites, resulting in increased active irinotecan metabolites in the blood [21]. According to the Clinical Pharmacogenetics Implementation Consortium (CPIC) guidelines, which provide genotype-guided pharmacotherapy recommendations, classify the UGT1A1 phenotype as follows: extensive metabolizer (EM), intermediate metabolizer (IM), and poor metabolizer (PM) [22,23]. Individuals who are heterozygous for a reduced function allele (e.g., having a UGT1A1*1/*28 genotype) are expected to be IMs, and those who are carriers of two reduced function alleles (e.g., having a UGT1A1*28/*28 genotype) are expected to be PMs. The US Food and Drug Administration (FDA)-approved product label for irinotecan recommends a reduced dose for patients homozygous for the UGT1A1*28 allele; however, the specific dose reduction for this patient population is not described. Because of uncertainty about clinical usefulness, a preemptive UGT1A1 test is not widely accepted.

Previous studies have not shown the impact of UGT1A1 polymorphisms, including the UGT1A1 PM phenotype, on FN development in PC patients receiving FOLFIRINOX. Therefore, in the present study, we investigated the risk factors for FN-in particular, UGT1A1 polymorphisms-in PC patients receiving FOLFIRINOX.

\section{Materials and Methods}

\subsection{Study Design}

We retrospectively reviewed the data of all patients diagnosed with PC, using the pancreatic cancer cohort registry of Severance Hospital, which is a prospective database for patients with PC treated with chemotherapy since 2015 [24].

The eligibility criteria were as follows: (1) $\geq 18$ years of age; (2) histologically or cytologically confirmed pancreatic adenocarcinoma; (3) at least one measurable or evaluable lesion 
following the Response Evaluation Criteria in Solid Tumors (RECIST), version 1.1 [25]; (4) Eastern Cooperative Oncology Group (ECOG) performance status (PS) $\leq 1$; (5) no previous anticancer treatment; (6) no FOLFIIRNOX dose reduction at the beginning; (7) no prophylactic pegfilgrastim or G-CSF; (7) available UGT1A1 genotype; and (8) adequate organ function (absolute neutrophil count $[\mathrm{ANC}] \geq 1500$ cells $/ \mu \mathrm{L}$, creatinine clearance $\geq 50 \mathrm{~mL} / \mathrm{min}$ ).

A total of 495 patients with PC who were treated with first line FOLFIRINOX between December 2017 and October 2020 were ascertained. We identified 154 patients who met the eligibility criteria. This study was approved by the Yonsei University Health System Institutional Review Board (approval number: 4-2020-1060) and conducted according to the principles of the Declaration of Helsinki.

\subsection{Chemotherapy Schedule and Response Evaluation}

The FOLFIRINOX regimen consisted of oxaliplatin $\left(85 \mathrm{mg} / \mathrm{m}^{2}\right)$ as a 2-h intravenous infusion (IVF), followed by leucovorin $\left(400 \mathrm{mg} / \mathrm{m}^{2}\right)$ administered as a 2-h IVF, and after $30 \mathrm{~min}$, the addition of irinotecan $\left(180 \mathrm{mg} / \mathrm{m}^{2}\right)$ given as $90-\mathrm{min}$ IVF, immediately followed by a $5-\mathrm{FU} 400 \mathrm{mg} / \mathrm{m}^{2}$ bolus and $2400 \mathrm{mg} / \mathrm{m}^{2}$ IVF for $46 \mathrm{~h}$, every 2 weeks.

At the start of treatment, the following tumor-related factors were studied and recorded: patient demographics; body mass index; date of diagnosis; tumor location; cancer stage; laboratory data, including levels of carbohydrate antigen (CA) 19-9; and UGT1A1 genotypes. Chemotherapy was postponed, or the dose was modified depending on the physician's decision, and treatment continued until the disease progressed, the toxicity was deemed to be unacceptable, or the patient refused treatment.

To assess treatment efficacy, computed tomography (CT) and serum CA 19-9 tests were conducted every 8 weeks. Treatment responses under the RECIST criteria were recorded by designated radiologists, and the attending physicians independently made the final judgment regarding treatment response.

\subsection{Assessment of Chemotherapy-Related Adverse Events}

To monitor for treatment-related AEs, the occurrence of AEs was thoroughly assessed by physicians and registered nurses at each visit during the course of chemotherapy. The severity grade of the AEs was recorded in the patients' medical records according to the Common Terminology Criteria for Adverse Events (CTCAE) version 5.0 [26]. The incidence of AEs was evaluated during the chemotherapy. The data were collected on 13 May 2021.

\subsection{Febrile Neutropenia and Grade 4 Neutropenia}

We defined FN as a single temperature of $\geq 38.3^{\circ} \mathrm{C}\left(101^{\circ} \mathrm{F}\right)$ or a temperature of $\geq 38.0^{\circ} \mathrm{C}\left(100.4{ }^{\circ} \mathrm{F}\right)$ sustained over an hour and an ANC $\leq 500$ cells $/ \mu \mathrm{L}$, as mentioned by the Infectious Disease Society of America (IDSA, 2010) guidelines [27]. Grade 4 neutropenia (NP grade 4 ) is defined as an ANC $<500$ cells $/ \mu \mathrm{L}$ according to CTCAE, version 5.0.

\subsection{UGT1A1 Polymorphisms}

To investigate the association between UGT1A1 polymorphisms and FN development, patients were divided into three groups based on the predicted UGT1A1 phenotype. An individual who has a heterozygous genotype for one decreased function allele (e.g., UGT1A1*1/*28 or UGT1A1*1/*6) was classified as an IM. A carrier of two decreased function alleles (e.g., UGT1A1*28/*28, UGT1A1*6/*6, or UGT1A1*6/*28 genotypes) was classified as a PM [23]. An individual carrying two normal function alleles (e.g., a UGT1A1*1/*1 genotype) was classified as an EM. All patients in this study were initially administered the original dose of FOLFIRINOX, regardless of the UGT1A1 genotype.

\subsection{Overall Survival and Progression-Free Survival}

The date of death and last follow-up were reviewed to estimate overall survival (OS) and progression-free survival (PFS). Survival and follow-up data were recorded until 
13 May 2021. The OS was computed from the date of diagnosis to the date of the last follow-up or death. PFS was calculated from the date of diagnosis to disease progression (or last follow-up or death). Living patients whose disease did not progress were censored at the date of the last follow-up.

\subsection{Statistical Analyses}

Baseline patient characteristics, laboratory data, treatment characteristics, and frequency of AEs were used to calculate descriptive statistics. The differences in baseline characteristics between the FN and non-FN groups were analyzed by using the Chi-squared test or Fisher's exact test for categorical variables, and the Student's $t$-test or Mann-Whitney test for continuous variables. For the comparison of the three groups according to UGT1A1 polymorphism, the Kruskal-Wallis test was performed, followed by the Mann-Whitney test with Bonferroni correction. A logistic progression model was used to estimate the odds ratio (OR) of potential risk factors for the occurrence of FN or NP grade 4. We calculated the median OS and PFS according to FN, using Kaplan-Meier curves, and compared these by using the log-rank test. Estimated medians with $95 \%$ confidence intervals (CIs) are reported. The Cox proportional hazard model was used to estimate the hazard ratio (HR) for FN or NP grade 4 events for each variable. Statistical significance was set at a two-tailed $p$-value of less than 0.05 for all tests. All analyses were conducted by using SPSS version 23.0 (SPSS, Chicago, IL, USA).

\section{Results}

\subsection{Patient Characteristics}

A total of 154 patients (31 in the FN group vs. 123 in the non-FN group) met the eligibility criteria. The baseline characteristics of the patients are shown in Table 1 . The median age of the patients was 62 years (interquartile range: 55-67 years), and 95 of the 154 patients $(61.7 \%)$ were male. A total of 55 of the 154 patients $(35.7 \%)$ had locally advanced PC, and 63 of the 154 (40.9\%) had metastatic PC at diagnosis. The other 36 of the 154 patients $(23.4 \%)$ were composed of 12 patients $(7.8 \%)$ with resectable PC, and 24 patients $(15.6 \%)$ with borderline resectable PC. Above them, eight $(66.7 \%)$ patients of resectable PC, and 16 (66.7\%) patients of borderline resectable PC were received surgical resection for curative aim. Of the 154 patients, 71 (46.1\%) were EMs, 66 (42.9\%) were IMs, and $17(11.0 \%)$ were PMs according to their UGT1A1 genotypes (Supplementary Table S1). Among the IMs, 31.2\% (48/154) had a UGT1A1*1/*6 genotype and $11.7 \%(18 / 54)$ had a UGT1A1*1/*28 genotype. Among the PMs, the UGT1A1*6/*28 genotype was the most prevalent $(7.1 \%, 11 / 154)$, followed by the UGT1A1*6/*6 $(3.2 \%, 5 / 154)$ and UGT1A1*28/*28 genotypes $(0.6 \%, 1 / 154)$. FN developed in 31 (20.1\%) patients, and NP grade 4 developed in $94(61.0 \%)$ patients; $54.8 \%(17 / 31)$ of the FN and $64.9 \%(61 / 94)$ of the NP grade 4 occurred within four chemotherapy cycles, respectively. 
Table 1. Baseline characteristics.

\begin{tabular}{|c|c|c|c|c|}
\hline Variable & $\begin{array}{l}\text { All Patients } \\
\quad(n=154)\end{array}$ & $\begin{array}{l}\text { FN Group } \\
(n=31)\end{array}$ & $\begin{array}{c}\text { Non-FN Group } \\
(n=123)\end{array}$ & $p$-Value \\
\hline \multicolumn{5}{|l|}{ Patient characteristics } \\
\hline Age, years & $62(55-67)$ & $66(62-70)$ & $60(55-66)$ & $<0.001$ \\
\hline \multicolumn{5}{|l|}{ Sex, no. $(\%)$} \\
\hline Male & $95(61.7)$ & $13(41.9)$ & $82(66.7)$ & 0.011 \\
\hline Female & $59(38.3)$ & $18(58.1)$ & $41(33.3)$ & \\
\hline \multicolumn{5}{|l|}{ ECOG PS } \\
\hline 0 & $130(84.4)$ & $21(67.7)$ & $109(88.6)$ & 0.010 \\
\hline 1 & $24(15.6)$ & $10(32.3)$ & $14(11.4)$ & \\
\hline BMI, median $\left(\mathrm{kg} / \mathrm{m}^{2}\right)$ & $22.7(21.2-25.0)$ & $23.6(21.2-26.1)$ & $22.3(21.2-24.9)$ & 0.499 \\
\hline $\mathrm{DM}$ & $50(32.5)$ & $13(41.9)$ & $37(30.1)$ & 0.208 \\
\hline \multicolumn{5}{|l|}{ UGT1A1 } \\
\hline Extensive metabolizer & $71(46.1)$ & $5(16.1)$ & $66(53.7)$ & $<0.001$ \\
\hline Intermediate metabolizer & $66(42.9)$ & $21(67.7)$ & $45(36.6)$ & \\
\hline Poor metabolizer & $17(11.0)$ & $5(16.1)$ & $12(9.8)$ & \\
\hline \multicolumn{5}{|l|}{ Tumor characteristics } \\
\hline \multicolumn{5}{|l|}{ Location } \\
\hline Head & $83(53.9)$ & $23(74.2)$ & $60(48.8)$ & 0.011 \\
\hline Body/Tail & $71(46.1)$ & $8(25.8)$ & $63(51.2)$ & \\
\hline \multicolumn{5}{|l|}{ Stage } \\
\hline Resectable & $12(7.8)$ & $3(9.7)$ & $9(7.3)$ & 0.449 \\
\hline Borderline Resectable & $24(15.6)$ & $2(6.5)$ & $22(17.9)$ & \\
\hline Locally advanced & $55(35.7)$ & $12(38.7)$ & $43(35.0)$ & \\
\hline Metastatic & $63(40.9)$ & $14(45.2)$ & $49(39.8)$ & \\
\hline \multicolumn{5}{|l|}{ Laboratory characteristics } \\
\hline WBC per $\mu \mathrm{L}$ & $6860.0(5565.0-8192.5)$ & $7590.0(5590.0-8660.0)$ & $6810.0(5550.0-7920.0)$ & 0.212 \\
\hline Neutrophils per $\mu \mathrm{L}$ & $4200.0(3172.5-5422.5)$ & $4600.0(3390.0-6490.0)$ & $4030.0(3150.0-5200.0)$ & 0.103 \\
\hline Lymphocytes per $\mu \mathrm{L}$ & $1630.0(1310.0-2040.0)$ & $1600.0(1140.0-2030.0)$ & $1630.0(1310.0-2060.0)$ & 0.442 \\
\hline NLR & $2.4(1.8-3.6)$ & $2.6(2.0-4.3)$ & $2.3(1.7-3.3)$ & 0.159 \\
\hline Hemoglobin, g/dL & $12.6(11.6-13.6)$ & $12.6(11.2-13.2)$ & $12.5(11.6-13.7)$ & 0.429 \\
\hline Platelets, $10^{3} / \mu \mathrm{L}$ & $242.5(198.8-316.6)$ & $273.0(220.0-346.0)$ & $228.0(196.0-308.0)$ & 0.033 \\
\hline Total bilirubin, $\mathrm{mg} / \mathrm{dL}$ & $0.6(0.5-1.0)$ & $0.8(0.6-2.0)$ & $0.6(0.4-0.9)$ & 0.002 \\
\hline AST, IU/L & $20.0(16.0-34.0)$ & $25.0(15.0-46.0)$ & $20.0(16.0-30.0)$ & 0.168 \\
\hline ALT, IU/L & $21.0(14.8-40.5)$ & $27.0(17.0-48.0)$ & $19.0(14.0-38.0)$ & 0.091 \\
\hline CA 19-9, U/mL & $245.0(28.5-1370.8)$ & $611.0(135.0-2364.0)$ & $209.0(20.8-1247.0)$ & 0.160 \\
\hline Albumin, $\mathrm{g} / \mathrm{dL}$ & $4.1(3.7-4.4)$ & $4.0(3.5-4.2)$ & $4.1(3.7-4.5)$ & 0.151 \\
\hline
\end{tabular}

Median (interquartile range) or $n(\%)$.

The proportion of female patients, as well as patients with poor ECOG PS, tumor location at the pancreas head, UGT1A1 IM, UGT1A1 PM, high platelet count, and total bilirubin level, was significantly higher in the FN group than in the non-FN group. The other variables were not statistically significantly different between the two groups.

The accumulated dose of irinotecan, oxaliplatin, 5-FU bolus, and continuous infusion of four cycles of chemotherapy was not statistically significantly different between the FN and non-FN groups (Table 2). The median time to FN development was the first four cycles. For this reason, cumulative doses in the non-FN group were computed for up to four cycles of chemotherapy. 
Table 2. Treatment characteristics preceding FN development.

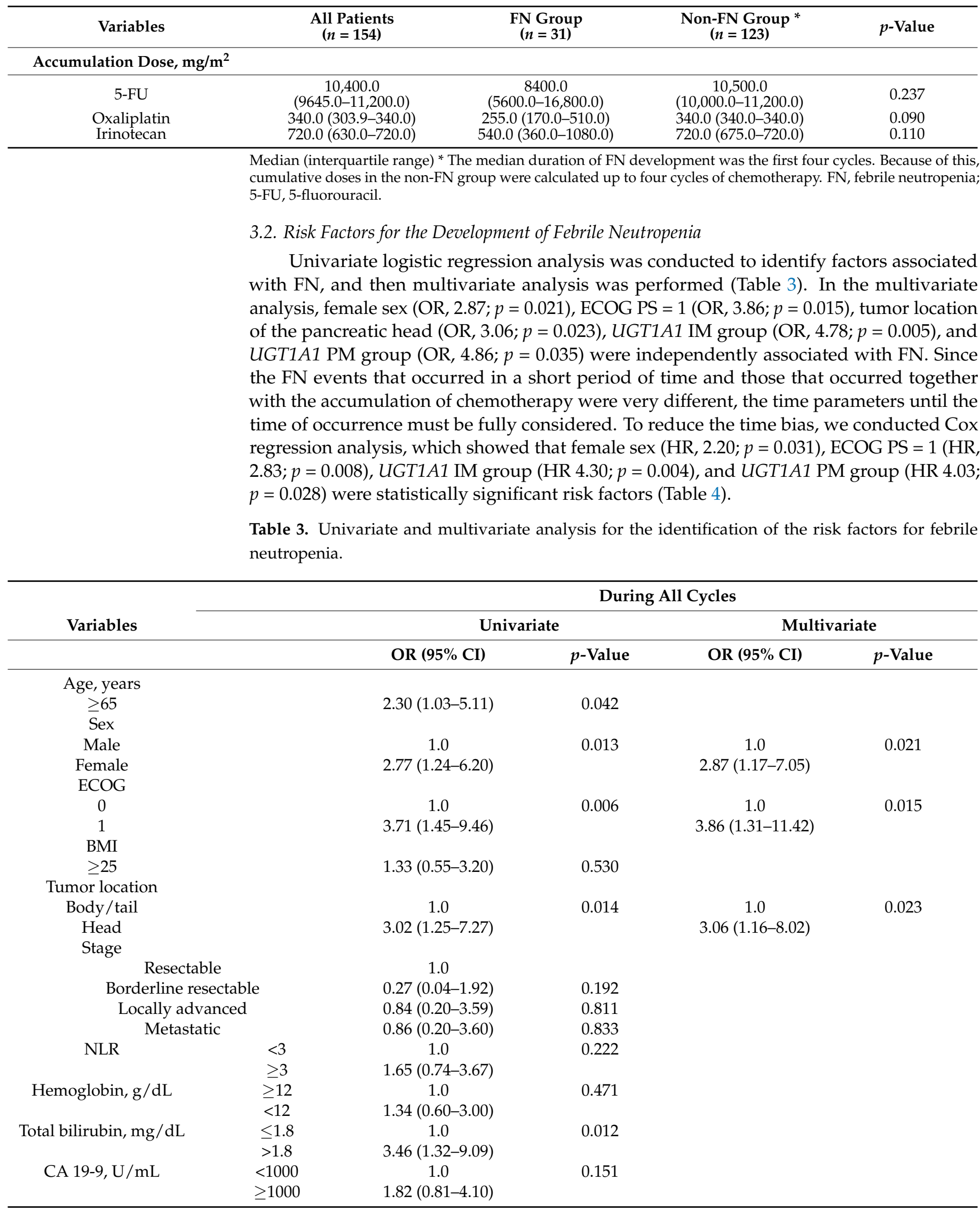


Table 3. Cont.

\begin{tabular}{ccccc}
\hline & & \multicolumn{2}{c}{ During All Cycles } \\
\cline { 2 - 5 } Variables & Univariate & \multicolumn{2}{c}{ Multivariate } \\
\hline & OR (95\% CI) & $p$-Value & OR (95\% CI) & $p$-Value \\
\hline $\begin{array}{c}\text { UGT1A1 phenotype } \\
\text { Extensive metabolizer } \\
\text { Intermediate } \\
\text { metabolizer }\end{array}$ & 1.0 & & 1.0 & \\
Poor metabolizer & $6.16(2.16-17.54)$ & 0.001 & $4.78(1.61-14.21)$ & 0.005 \\
\hline
\end{tabular}

BMI, body mass index; CA, carbohydrate antigen; CI, confidence interval; ECOG PS, Eastern Cooperative Oncology Group performance status; NLR, neutrophil to lymphocyte ratio; OR, odds ratio.

Table 4. Multivariate analysis using Cox regression analysis to identify risk factors for febrile neutropenia.

\begin{tabular}{cccccc}
\hline \multirow{2}{*}{ Variable } & \multicolumn{5}{c}{ Febrile Neutropenia } \\
\cline { 2 - 6 } & Unadjusted HR & $\boldsymbol{p}$-Value & Adjusted HR & $\mathbf{9 5 \%}$ CI & $p$-Value \\
\hline Female sex & 2.40 & 0.016 & 2.20 & $1.07-4.51$ & 0.031 \\
ECOG PS = & 3.29 & 0.002 & 2.83 & $1.32-6.10$ & 0.008 \\
UGT1A1 IM & 5.15 & 0.001 & 4.30 & $1.61-11.52$ & 0.004 \\
UGT1A1 PM & 4.49 & 0.018 & 4.03 & $1.16-14.01$ & 0.028 \\
\hline
\end{tabular}

CI, confidence interval; ECOG PS, Eastern Cooperative Oncology Group performance status; HR, hazard ratio IM, intermediate metabolizer; PM, poor metabolizer; UGT1A1, uridine diphospho-glucuronosyltransferase 1A1.

\subsection{Risk Factors for the Development of Grade 4 Neutropenia}

Univariate logistic regression analysis was conducted to identify factors associated with NP grade 4 , and multivariable analysis was performed (Table 5). In multivariate analysis, female sex (OR, 3.44; $p=0.001)$ and the UGT1A1 PM group (OR, 4.06; $p=0.044)$ were independently associated with NP grade 4 . When the Cox regression analysis was applied with the concept of time, female sex $(\mathrm{HR}, 1.98 ; p=0.001)$ was found to be a significant risk factor (Table 6).

We included UGT1A1 IM group in the multivariate analysis in spite of statistical insignificance $(p=0.231)$, because previous studies showed that IM group was related to neutropenia.

\subsection{Relationship between UGT1A1 Phenotypes and Hematologic Toxicities}

We investigated the association between UGT1A1 phenotypes and serious hematologic AEs (Grade III/IV). Table 7 shows that there were significant differences in the aspects of anemia (EMs $4.2 \%$ vs. IMs $19.7 \%$ and PMs $23.5 \%, p=0.011$ ) and FN (EMs $7.0 \%$ vs. IMs $31.8 \%$ and PMs $29.4 \%, p=0.001$ ) among the three groups of UGT1A1 phenotypes. The UGT1A1 IM and PM groups tended to be susceptible to NP grade 4, although the differences were not statistically significant.

We further analyzed the impact of UGT1A1 polymorphisms on diarrhea in Table 8. Neither diarrhea nor severe diarrhea (Grade III/IV) showed a significant association with the UGT1A1 polymorphism. 
Table 5. Univariate and multivariate analysis related to grade 4 neutropenia.

\begin{tabular}{|c|c|c|c|c|c|}
\hline \multirow{3}{*}{ Variable } & & \multicolumn{4}{|c|}{ During All Cycles } \\
\hline & & \multicolumn{2}{|c|}{ Univariate } & \multicolumn{2}{|c|}{ Multivariate } \\
\hline & & OR $(95 \%$ CI $)$ & $p$-Value & OR $(95 \% \mathrm{CI})$ & $p$-Value \\
\hline \multicolumn{6}{|l|}{ Age, years } \\
\hline$\geq 65$ & & $1.95(0.96-3.94)$ & 0.063 & & \\
\hline \multicolumn{6}{|c|}{ (10. } \\
\hline Male & & 1.0 & 0.001 & 1.0 & 0.001 \\
\hline Female & & $3.47(1.66-7.23)$ & & $3.44(1.63-7.25)$ & \\
\hline \multicolumn{6}{|l|}{ ECOG } \\
\hline 0 & & 1.0 & 0.767 & & \\
\hline 1 & & $0.88(0.63-2.12)$ & & & \\
\hline \multicolumn{6}{|l|}{ BMI } \\
\hline$\geq 25$ & & $0.76(0.53-2.40)$ & 1.127 & & \\
\hline \multicolumn{6}{|l|}{ Tumor location } \\
\hline Body/tail & & 1.0 & 0.152 & & \\
\hline Head & & $1.61(0.84-3.10)$ & & & \\
\hline \multicolumn{6}{|l|}{ Stage } \\
\hline \multicolumn{2}{|c|}{ Resectable } & 1.0 & & & \\
\hline \multicolumn{2}{|c|}{ Borderline resectable } & $1.66(0.41-6.71)$ & 0.481 & & \\
\hline \multicolumn{2}{|c|}{ Locally advanced } & $3.13(0.87-11.28)$ & 0.081 & & \\
\hline \multicolumn{2}{|c|}{ Metastatic } & $2.13(0.61-7.46)$ & 0.238 & & \\
\hline \multirow[t]{2}{*}{ NLR } & $<3$ & 1.0 & 0.376 & & \\
\hline & $\geq 3$ & $0.74(0.38-1.45)$ & & & \\
\hline \multirow[t]{2}{*}{ Hemoglobin, g/dL } & $\geq 12$ & 1.0 & 0.950 & & \\
\hline & $<12$ & $0.98(0.50-1.92)$ & & & \\
\hline \multirow[t]{2}{*}{$\begin{array}{l}\text { Total bilirubin, } \\
\text { mg/dL }\end{array}$} & $\leq 1.8$ & 1.0 & 0.787 & & \\
\hline & $>1.8$ & $1.14(0.45-2.90)$ & & & \\
\hline \multirow[t]{2}{*}{ CA 19-9, U/mL } & $<1000$ & 1.0 & 0.188 & & \\
\hline & $\geq 1000$ & $1.62(0.79-3.34)$ & & & \\
\hline \multicolumn{6}{|c|}{ UGT1A1 phenotype } \\
\hline \multicolumn{2}{|c|}{ Extensive metabolizer } & 1.0 & & 1.0 & \\
\hline \multicolumn{2}{|c|}{ Intermediate metabolizer } & $1.52(0.77-3.01)$ & 0.231 & $1.41(0.69-2.88)$ & 0.343 \\
\hline \multicolumn{2}{|c|}{ Poor metabolizer } & $4.05(1.07-15.34)$ & 0.039 & $4.06(1.04-15.87)$ & 0.044 \\
\hline
\end{tabular}

BMI, body mass index; CI, confidence interval; ECOG PS, Eastern Cooperative Oncology Group performance status; NLR, neutrophil to lymphocyte ratio; OR, odds ratio.

Table 6. Multivariate analysis using Cox regression analysis to identify risk factors for grade 4 neutropenia.

\begin{tabular}{cccccc}
\hline \multirow{2}{*}{ Variable } & \multicolumn{5}{c}{ Grade 4 Neutropenia } \\
\cline { 2 - 6 } & Unadjusted HR & $\boldsymbol{p}$-Value & Adjusted HR & $\mathbf{9 5 \%}$ CI & \multicolumn{1}{c}{$1.31-2.98$} \\
Female sex & 2.06 & 0.001 & 1.98 & $0.83-2.00$ & 0.001 \\
UGT1A1 IM & 1.32 & 0.217 & 1.29 & $0.96-3.33$ & 0.066 \\
UGT1A1 PM & 2.01 & 0.026 & 1.79 & 0.964 \\
\hline
\end{tabular}

CI, confidence interval; HR, hazard ratio; IM, intermediate metabolizer; PM, poor metabolizer; UGT1A1, uridine diphospho-glucuronosyltransferase 1A1.

We investigated the baseline characteristics among the three UGT1A1 phenotype groups, including chemotherapy characteristics, to rule out selection bias (Supplementary Tables S2 and S3). The total bilirubin before chemotherapy was significantly higher in PMs, compared with EMs and IMs (PMs 1.2 vs. EMs 0.5 and IMs 0.6, $p<0.001$ ), although the other variables showed no differences. 
Table 7. Serious hematologic toxicity (Grade III/IV) according to UGT1A1 phenotype.

\begin{tabular}{cccccc}
\hline & $\begin{array}{c}\text { Extensive } \\
\text { Metabolizer } \\
(\boldsymbol{n}=\mathbf{7 1})\end{array}$ & $\begin{array}{c}\text { Intermediate } \\
\text { Metabolizer } \\
(\boldsymbol{n}=\mathbf{6 6})\end{array}$ & $\begin{array}{c}\text { Poor Metabolizer } \\
(\boldsymbol{n}=\mathbf{1 7})\end{array}$ & $\begin{array}{c}\text { All Patients } \\
(\boldsymbol{n}=\mathbf{1 5 4})\end{array}$ & $\boldsymbol{p}$-Value \\
\hline Neutropenia & $57 / 71(80.3)$ & $58 / 66(87.9)$ & $15 / 17(88.2)$ & $130 / 154(84.4)$ & 0.411 \\
Neutropenia (Grade IV) & $38 / 71(53.5)$ & $42 / 66(63.6)$ & $14 / 17(82.4)$ & $94 / 154(61.0)$ & 0.082 \\
Febrile neutropenia & $5 / 71(7.0)$ & $21 / 66(31.8)$ & $5 / 17(29.4)$ & $31 / 154(20.1)$ & 0.001 \\
Anemia & $3 / 71(4.2)$ & $13 / 66(19.7)$ & $4 / 17(23.5)$ & $20 / 154(13.0)$ & 0.011 \\
Thrombocytopenia & $4 / 71(5.6)$ & $10 / 66(15.2)$ & $3 / 17(17.6)$ & $17 / 154(11.0)$ & 0.118 \\
\hline
\end{tabular}

Data are presented as number of patients/total number (\%).

Table 8. Incidence of diarrhea according to UGT1A1 phenotype.

\begin{tabular}{cccccc}
\hline & $\begin{array}{c}\text { Extensive } \\
\text { Metabolizer } \\
(\boldsymbol{n}=\mathbf{7 1})\end{array}$ & $\begin{array}{c}\text { Intermediate } \\
\text { Metabolizer } \\
(\boldsymbol{n}=\mathbf{6 6})\end{array}$ & $\begin{array}{c}\text { Poor Metabolizer } \\
(\boldsymbol{n}=\mathbf{1 7})\end{array}$ & $\begin{array}{c}\text { All Patients } \\
(\boldsymbol{n}=\mathbf{1 5 4})\end{array}$ & $\boldsymbol{p}$-Value \\
\hline Diarrhea & $22 / 71(31.0)$ & $21 / 66(31.8)$ & $4 / 17(23.5)$ & $47 / 154(30.5)$ & 0.801 \\
Diarrhea (Grade III/ IV) & $2 / 71(2.8)$ & $5 / 66(7.6)$ & $1 / 17(5.9)$ & $8 / 154(5.2)$ & 0.427 \\
\hline
\end{tabular}

Data are presented as number of patients/total number $(\%)$.

\subsection{Overall Survival and Progression-Free Survival}

The median follow-up period was 11.2 months (interquartile range 7.5-15.9), and the median number of chemotherapy cycles per patient was 11 (interquartile range 6.0-18.3). During this period, 43 patients $(27.9 \%)$ died, and 89 patients $(57.8 \%)$ experienced disease progression.

Our analysis of the treatment outcomes according to FN development showed that the FN group exerted a significant unfavorable effect on OS (18.7 months vs. 24.3 months; $\log$-rank $p=0.007$ ) than the non-FN group (Figure 1a). Of the 31 patients in the FN group, seven $(22.6 \%)$ experienced septic shock, and one patient died due to FN-related septic shock. However, the FN group did not have a significant effect on PFS (Figure 1b).

We compared the relative dose intensity (RDI) of FOLFIRINOX between the FN and non-FN groups to determine the reason for the difference in OS. The FN group received a lower dose of the FOLFIRINOX regimen than the non-FN group (Supplementary Table S4). The median RDI of irinotecan, oxaliplatin, and 5-FU (infusion) was significantly lower in the FN group. In contrast, the median duration of chemotherapy did not differ between the two groups.

The analysis revealed that the treatment outcomes according to the UGT1A1 phenotype did not have a significant effect on OS and PFS (Figure 2a,b).
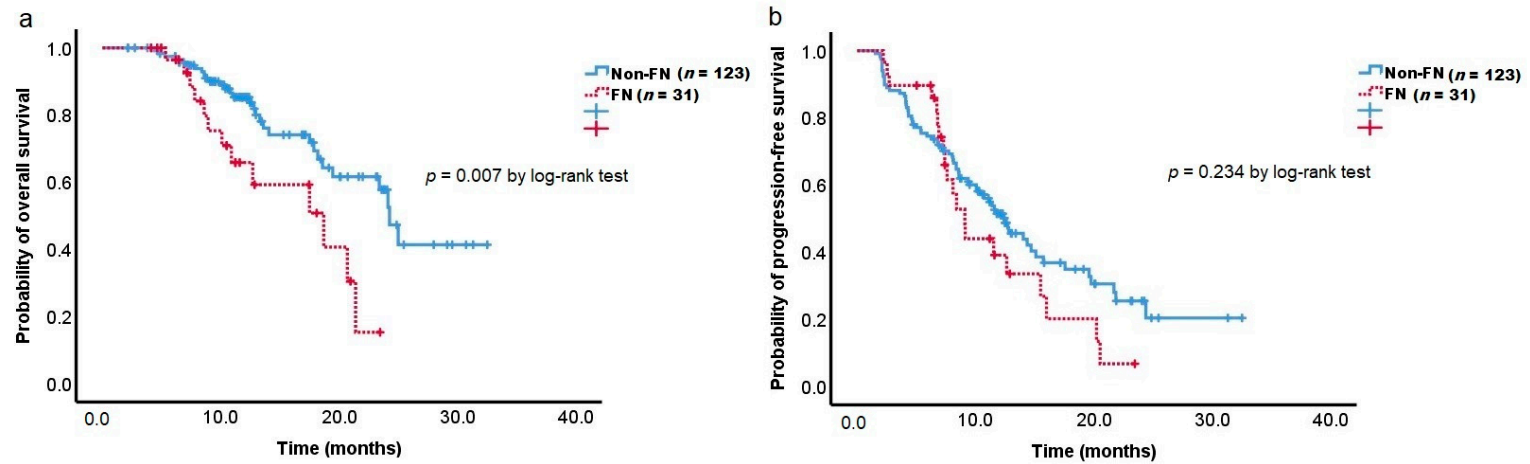

Figure 1. Kaplan-Meier estimates of overall survival (a) and progression-free survival (b) according to febrile neutropenia. 

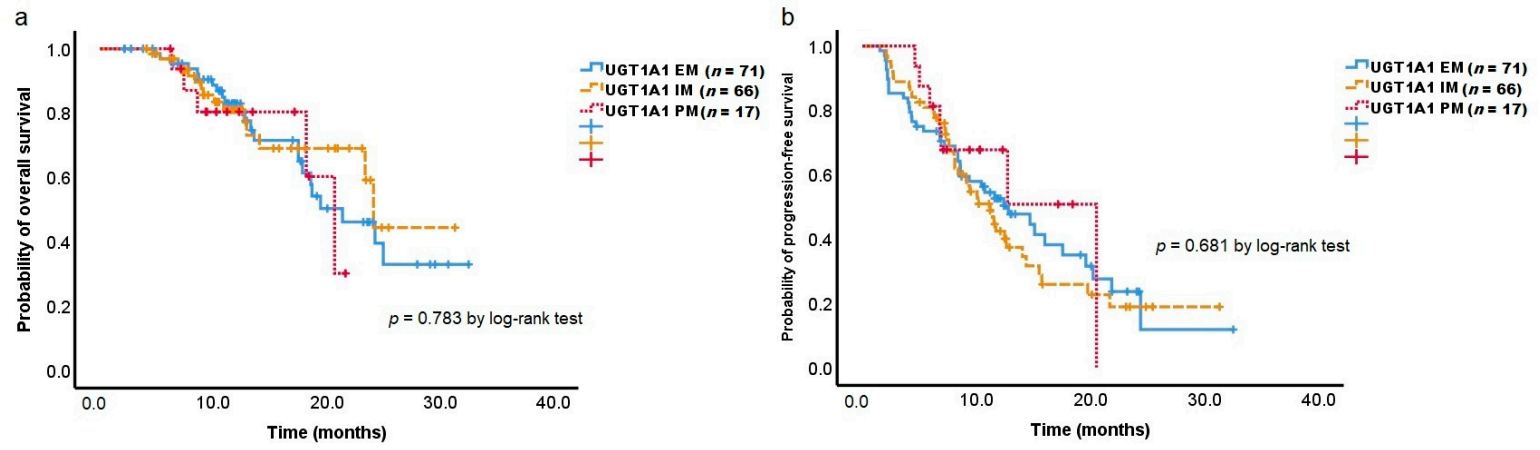

Figure 2. Kaplan-Meier estimates of overall survival (a) and progression-free survival (b) according to UGT1A1 phenotype.

\section{Discussion}

The FOLFIRINOX regimen is often limited because of the high incidence of FN. In this study, FN developed in 31 (20.1\%) patients, and NP grade 4 developed in $94(61.0 \%)$ patients. Similarly, previous studies reported FN and grade $3 / 4$ NP in $5.4-22.2 \%$ and $45.7-77.8 \%$ of patients, respectively $[2,5,6]$. We identified female sex, ECOG PS $=1$, UGT1A1 IM, and UGT1A1 PM as risk factors for FN in PC patients receiving FOLFIRINOX. Among them, the HR of UGT1A1 status was the highest.

There have been few studies on the impact of UGT1A1 polymorphisms on FN development in PC patients treated with FOLFIRINOX. Most studies on the interaction of irinotecan with UGT1A1 have focused on patients with colorectal cancer (CRC) receiving the FOLFIRI (irinotecan, fluorouracil, and leucovorin) regimen [28-32]. Some guidelines recommend genotyping for UGT1A1 and considering at least a $25 \%$ reduction in irinotecan for patients homozygous for UGT1A1*28 [33,34]. However, UGT1A1 genotyping is not routinely performed before the start of irinotecan-containing chemotherapy because of the scarcity of large prospective studies [35].

FOLFIRINOX, which adds oxaliplatin over the FOLFIRI regimen, should be evaluated for the impact of UGT1A1 on PC patients with poorer prognosis than CRC patients. In a multicenter observational study of 199 PC patients in Japan, patients heterozygous for UGT1A1 were reported to suffer more severe diarrhea than those with the wild-type genotype when receiving the original dose of FOLFIRINOX [36]. In a retrospective study of 106 PC patients treated with FOLFIRINOX in a single-center, female sex, overweight, and biliary stent insertion were reported as risk factors for FN development [11]. Since our study did not perform a multivariate analysis of UGT1A1, we could only identify risk factors for the clinical characteristics of patients. In a prospective study of patients with advanced gastrointestinal cancer, including PC, UGT1A1 genotyping-guided modified FOLFIRINOX dose failed to demonstrate tolerability to PC patients [37]. In contrast, in a prospective study of 50 patients with advanced gastrointestinal cancer including 30 patients with PC, UGT1A1-genotyping-guided dose modification of FOLFIRABRAX (irinotecan, fluorouracil, leucovorin, and nab-paclitaxel) with prophylactic pegfilgrastim was tolerable in patients with UGT1A1 wild type or UGT1A1*1/*28 genotypes. However, this study evaluated the dose-limiting toxicity (DLT) rate in the first cycle only, and the impact on the entire period of chemotherapy was unknown [38].

In this study, we investigated the risk factors for FN development through multivariate analysis, including the genetic factor of UGT1A1 status, during the entire chemotherapy period after receiving FOLFIRINOX. UGT1A1 was found to be a more powerful risk factor than the clinical features of patients. To the best of our knowledge, this study is the first to analyze risk factors for FN in PC patients receiving FOLFIRINOX as first-line treatment, including patients' clinical features and UGT1A1 status.

In the present study, PMs for the UGT1A1 gene were found in $11.0 \%$ of PC patients, with the UGT1A1*6/*28 genotype being the most prevalent $(7.1 \%)$, followed by 
UGT1A1*6/*6 $(3.2 \%)$ and UGT1A1*28/*28 (0.6\%). IMs for the UGT1A1 gene were found in $42.9 \%$ of patients, with the UGT1A1*1/ ${ }^{*} 6$ genotype being the most common $(31.2 \%)$, followed by UGT1A1*1/*28 (11.7\%). EMs were found in $46.1 \%$ of patients. Our data are generally in accordance with those reported by the CPIC guidelines, which showed that the prevalence of PMs, IMs, and EMs is $8 \%, 42 \%$, and 50\%, respectively, in East Asians [22].

UGT1A1*28 and UGT1A1*6 are well-studied UGT1A1 polymorphisms with respect to irinotecan pharmacokinetics and pharmacodynamics. Especially among Caucasian patients, UGT1A1*28 appears to be a strong predictor of NP. However, in Asian populations, the UGT1A1*6 variant is more common and seems to be a more precise predictor of NP. $[39,40]$ In this study population, UGT1A1*6, with a minor allele frequency of $22.4 \%$, was more common than UGT1A1*28, with a minor allele frequency of $10.1 \%$, which is generally consistent with the Korean Reference Genome database [41].

Several studies have shown an association between the degree of systemic exposure to SN-38 and the risk of neutropenia. Minami et al. reported the pharmacokinetics of irinotecan in patients with or without UGT1A1*6 or $* 28$ in 177 Japanese cancer patients, in which the area under the concentration curve ratio of SN-38G to SN-38 was lower in patients with one and two haplotypes harboring ${ }^{*} 6$ or ${ }^{*} 28$ than in those without $* 6$ or $* 28$, and lowest in patients with two haplotypes harboring * 6 or *28 [42].

Most studies support a "gene-drug exposure" interaction, in which toxicities among UGT1A1 polymorphism carriers were associated with higher levels of exposure to irinotecan [43]. The highest correlations with severe NP and diarrhea were found in UGT1A1*28 homozygotes with irinotecan doses of $180 \mathrm{mg} / \mathrm{m}^{2}$, especially at doses of $250 \mathrm{mg} / \mathrm{m}^{2}[34,43,44]$. The dose of irinotecan, which may increase the risk of toxicity to UGT1A1 IMs, has not been fully determined. A trial using genotype-guided dosing of irinotecan in CRC patients receiving FOLFIRI regimen found that the ${ }^{*} 1 /{ }^{*} 1$ and ${ }^{*} 1 /{ }^{*} 28$ genotypes could tolerate higher than the recommended $180 \mathrm{mg} / \mathrm{m}^{2}$ every 2 weeks [29]. However, many existing studies have specifically investigated CRC, and there are insufficient studies on the toxicity and efficacy of the FOLFIRINOX regimen associated with UGT1A1 polymorphisms in PC patients.

A retrospective study of 31 PC patients with the UGT1A1 PM phenotype reported that an initial dose of irinotecan $\leq 120 \mathrm{mg} / \mathrm{m}^{2}$ reduced NP grade $4(20 \% ; 5 / 24)$ more than an initial dose of irinotecan $\geq 150 \mathrm{mg} / \mathrm{m}^{2}(67 \% ; 4 / 6)$ [45]. A response rate of $21.4 \%$, a median PFS of 8.1 months, and a median OS of 15.8 months in patients given irinotecan at an initial dose of $\leq 120 \mathrm{mg} / \mathrm{m}^{2}$ were not different from previous studies. However, the authors concluded that a prospective study is needed to determine the RDI for validating the AEs and efficacy of FOLFIRINOX for UGT1A1 PM, because various doses of irinotecan were administered, and each group of patients divided by the different doses of irinotecan rendered only a few cases. In the present study, the median RDI of irinotecan in the FN group was $88 \%$ of the standard dose, and the median RDI of the UGT1A1 PM group was $89 \%$, indicating a higher dose than in previous studies. Future studies are needed to determine the dose of irinotecan according to the high-risk features of FN and UGT1A1 polymorphisms.

A few studies have started FOLFIRINOX at the original dose for PC patients with UGT1A1 PM; therefore, the present study is considered to be the first report on the incidence of AEs. Of the 17 patients in the UGT1A1 PM group, 88.2\% experienced serious (grade 3 or 4) NP, and 29.4\% experienced FN, far more than in the PRODIGE4/ ACCORD11 trial (45.7\%, and $5.4 \%$, respectively). Likewise, the incidence of serious anemia and thrombocytopenia was higher than that in a previous study (23.5\% vs. $7.8 \%$ and $17.6 \%$ vs. $9.1 \%$, respectively).

In addition to genetic factors, female sex and ECOG PS $=1$ were risk factors for FN development. In our previous study, female sex was an independent risk factor for FN occurrence and ECOG PS = 1 was not statistically significant but showed a tendency for vulnerability to FN, which was generally consistent with the results of this study [11]. Innocenti et al. reported that females tended to be more prone to NP when irinotecan was administered to patients with CRC or lymphoma [16,46]. Although the mechanism by which females are more likely to develop FN has not been elucidated, Crawford et al. 
suggested that differences in total bone mass might cause the higher incidence of FN observed in females [47] because lower bone-marrow volume might be associated with lower tolerance to chemotherapy. Four studies in patients with lymphoma, breast cancer, and lung cancer have shown poor performance as risk factors for FN development [48]. Additionally, it has been suggested that physiological age, as indicated by ECOG PS, may serve as a risk factor for chronological age in older patients.

FN often results in severe infections, longer hospital stays, life-threatening morbidity, and mortality. Patients may also lose potential opportunities for treatment because of the severe consequences of FN. In the present study, seven patients (22.6\%) in 31 of the FN group experienced septic shock, and one patient died due to FN-related septic shock. This patient had the UGT1A1*6/*6 genotype. Four patients in six of the non-mortality cases had UGT1A1*1/*6 genotypes, and two patients had the UGT1A1*1/*1 genotype. Moreover, there was a significant difference in OS between the FN and non-FN groups (median OS, 18.7 months vs. 24.3 months, $\log$-rank $p=0.007)$. However, there was no difference in median PFS between the two groups. At present, we do not know the precise reason for the difference between OS and PFS. This might be related to the fact that six of the 31 patients in the FN group suffered from severe $\mathrm{FN}$ in which chemotherapy was delayed for more than a week, and one died from FN-related infections. A retrospective study of 165 PC patients treated with FOLFIRINOX reported that prophylactic primary G-CSF usage reduced the risk of NP $(55.6 \%$ to $31.6 \% ; p=0.003)$ and $\mathrm{FN}(18.5 \%$ to $1.8 \% ; p=0.002)$ and improved OS (8.8 to 14.7 months; HR: $1.766, p=0.001$ ) [49]. The author explained that an increase in RDI and treatment duration of FOLFIRINOX could result in a positive impact on survival. Similarly, there was a decrease in the median RDI of each chemotherapeutic agent except 5 -FU (bolus) in the FN group compared with the non-FN group. However, there was no difference in the median number of chemotherapy cycles between the two groups. The causal relationship between reduced RDI and negative impact on OS could not be assessed due to the retrospective design of this study. It is necessary to confirm this finding in large prospective studies.

Regarding the association between UGT1A1 and OS or PFS, there was no difference among UGT1A1 status. No previous study has evaluated the influence of UGT1A1 polymorphisms on survival in PC patients receiving FOLFIRINOX. CRC patients with one or more UGT1A1*28 alleles did not show significant results for OS and PFS [30,50]. Conflicting results also exist. In a prospective study, 250 metastatic CRC patients were treated with the FOLFIRI regimen as a first-line treatment. It suggested no significant survival benefit but revealed a positive effect on tumor response in patients with the UGT1A1 PM phenotype. The higher response rates were explained by higher SN-38 concentrations in these patients [28]. Further studies are needed regarding the association between UGT1A1 polymorphisms and therapeutic outcomes in PC patients receiving FOLFIRINOX.

This study had some limitations. First, according to previous studies on pharmacokinetics data of irinotecan, UGT1A1 PMs were expected to experience higher rates of AEs than UGT1A1 IMs; however, in this study, the HR on FN did not differ from that of IMs. This is possibly because the number of UGT1A1 PMs was smaller than that of IMs and EMs. Second, additional irinotecan pharmacokinetics data are needed to confirm the negative effect of UGT1A1 polymorphisms on lower survival in the FN group. Depending on the actual exposure to SN-38, evidence of modified FOLFIRINOX by genotype-guided dosing and the use of prophylactic G-CSF may be established in the high-risk group for FN development. Third, we could not evaluate genetic variants other than UGT1A1. It has been suggested that variations in the genes encoding CYP3A4, SLCO1B1, and ABC transporters, as well as other UGT1A enzymes, are related to the pharmacokinetics and pharmacodynamics of irinotecan [22,46]. With respect to fluorouracil, DPD, TYMS, DPYS, CDA, and MTHFR have previously been reported to be associated with toxicity. It should be considered that these polymorphisms exist at lower frequencies among Asians, and the patient population in this study consisted of East Asians [51-53]. Since the use of next-generation sequencing and gene panel tests will increase in the future, additional 
studies on the association of the pharmacokinetics of FOLFIRINOX with variants in genes other than UGT1A are needed. Fourth, our results might not be applicable to the Western population, because $U G T 1 A 1^{*} 6$ is predominantly found in the Asian population. In our study, among the seven serious FN events, five patients had more than one UGT1A1*6 allele. Of the $31 \mathrm{FN}$ cases, 21 patients had one or more UGT1A1*6 alleles, while only seven patients had one or more UGT1A1*28 alleles. Fifth, for the application of real-world practice, it is necessary to evaluate the toxicity and efficacy of UGT1A1 genotype-guided dosing of FOLFIRINOX through a large-scale prospective study. Sixth, in this study, neither diarrhea nor severe diarrhea (grade III/IV) demonstrated a significant association with UGT1A1 polymorphism. It differed from earlier studies that reported that more diarrhea in the UGT1A1 IM and PM groups, compared to the EM group, was probably the result of the small sample size. Retrospective analysis could also influence this outcome. Seventh, we tried to analyze the association of recent surgery ( $\leq 1$ month before chemotherapy start) and FN. However, of 24 patients who underwent surgery, only one of them was indicated as having had recent surgery, so an analysis could not be performed. Recently, FOLFIRINOX has been administered as adjuvant chemotherapy for PC patients after surgery, so further research on the association between surgery and FN is needed.

\section{Conclusions}

We investigated the polyfactorial basis of FN, a common adverse effect of the FOLFIRINOX regimen. To our knowledge, a comprehensive investigation such as this has never been conducted, and we propose that UGT1A1 is the strongest predictive factor for FN and that this gene should be screened prior to chemotherapy. Further studies are necessary to establish definitive guidelines for the dose reduction of FOLFIRINOX and prophylactic G-CSF usage in PC patients with UGT1A1 polymorphisms.

Supplementary Materials: The following supporting information can be downloaded at https: / / www.mdpi.com/article/10.3390/cancers14051244/s1. Supplementary Table S1: Allele frequency for UGT1A1*6 and UGT1A1*28 in 154 patients who received FOLFIRINOX for pancreatic cancer. Supplementary Table S2: Baseline characteristics according to UGT1A1 phenotype. Supplementary Table S3: Treatment characteristics according to UGT1A1 phenotype. Supplementary Table S4: Treatment characteristics during entire chemotherapy period.

Author Contributions: Conceptualization, J.K. and S.B.; Data curation, J.K., H.S.L., J.H.J., M.J.C., J.Y.P., S.W.P., S.Y.S. and S.B.; Formal analysis, J.K.; Investigation, J.K.; Methodology, J.K. and S.B.; Project administration, S.B.; Resources, S.B.; Software, J.K.; Supervision, S.B.; Validation, S.B.; Writingoriginal draft, J.K.; Writing-review \& editing, J.K. and S.B. All authors have read and agreed to the published version of the manuscript.

Funding: This study was supported by a grant of the Korea Health Technology R\&D Project through the Korea Health Industry Development Institute, funded by the Ministry of Health and Welfare, Republic of Korea (grant number: HI19C064201).

Institutional Review Board Statement: The study was conducted according to the guidelines of the Declaration of Helsinki and approved by the Institutional Review Board of Yonsei University on 9 November 2020 (approval number: 4-2020-1060).

Informed Consent Statement: Informed consent was obtained from all subjects involved in the study.

Data Availability Statement: The data presented in this study are available in this article.

Conflicts of Interest: The authors declare no conflict of interest.

\section{References}

1. Siegel, R.L.; Miller, K.D.; Jemal, A. Cancer statistics, 2017. CA Cancer J. Clin. 2017, 67, 7-30. [CrossRef] [PubMed]

2. Conroy, T.; Desseigne, F.; Ychou, M.; Bouché, O.; Guimbaud, R.; Bécouarn, Y.; Adenis, A.; Raoul, J.L.; Gourgou-Bourgade, S.; de la Fouchardière, C.; et al. Folfirinox versus gemcitabine for metastatic pancreatic cancer. N. Engl. J. Med. 2011, 364, $1817-1825$. [CrossRef] 
3. Sohal, D.P.S.; Mangu, P.B.; Khorana, A.A.; Shah, M.A.; Philip, P.A.; O’Reilly, E.M.; Uronis, H.E.; Ramanathan, R.K.; Crane, C.H.; Engebretson, A.; et al. Metastatic pancreatic cancer: American society of clinical oncology clinical practice guideline. J. Clin. Oncol. 2016, 34, 2784-2796. [CrossRef]

4. Tempero, M.A.; Malafa, M.P.; Al-Hawary, M.; Behrman, S.W.; Benson, A.B.; Cardin, D.B.; Chiorean, E.G.; Chung, V.; Czito, B.; Del Chiaro, M.; et al. Pancreatic adenocarcinoma, version 2.2021, nccn clinical practice guidelines in oncology. J. Natl. Compr. Cancer Netw. 2021, 19, 439-457. [CrossRef]

5. Okusaka, T.; Ikeda, M.; Fukutomi, A.; Ioka, T.; Furuse, J.; Ohkawa, S.; Isayama, H.; Boku, N. Phase ii study of FOLFIRINOX for chemotherapy-naïve Japanese patients with metastatic pancreatic cancer. Cancer Sci. 2014, 105, 1321-1326. [CrossRef]

6. Hosein, P.J.; Macintyre, J.; Kawamura, C.; Maldonado, J.C.; Ernani, V.; Loaiza-Bonilla, A.; Narayanan, G.; Ribeiro, A.; Portelance, L.; Merchan, J.R.; et al. A retrospective study of neoadjuvant FOLFIRINOX in unresectable or borderline-resectable locally advanced pancreatic adenocarcinoma. BMC Cancer 2012, 12, 199. [CrossRef]

7. Kang, H.; Jo, J.H.; Lee, H.S.; Chung, M.J.; Bang, S.; Park, S.W.; Song, S.Y.; Park, J.Y. Comparison of efficacy and safety between standard-dose and modified-dose FOLFIRINOX as a first-line treatment of pancreatic cancer. World J. Gastrointest. Oncol. 2018, 10, 421-430. [CrossRef]

8. Cho, I.R.; Kang, H.; Jo, J.H.; Lee, H.S.; Chung, M.J.; Park, J.Y.; Park, S.W.; Song, S.Y.; An, C.; Park, M.S.; et al. Folfirinox vs gemcitabine/nab-paclitaxel for treatment of metastatic pancreatic cancer: Single-center cohort study. World J. Gastrointest. Oncol. 2020, 12, 182-194. [CrossRef] [PubMed]

9. National Comprehensive Cancer Network. Hematopoietic Growth Factors, Version 4. 2021. Available online: https://www.nccn. org/professionals/physician_gls/pdf/growthfactors.pdf (accessed on 5 October 2021).

10. Sasaki, M.; Ueno, H.; Kuchiba, A.; Koga, F.; Shiba, S.; Sakamoto, Y.; Kondo, S.; Morizane, C.; Okusaka, T. P-167risk factors for febrile neutropenia in patients with unresectable pancreatic cancer receiving FOLFIRINOX as the first-line treatment. Ann. Oncol. 2015, 26, iv48. [CrossRef]

11. Keum, J.; Lee, H.S.; Kang, H.; Jo, J.H.; Chung, M.J.; Park, J.Y.; Park, S.W.; Song, S.Y.; Bang, S. Single-center risk factor analysis for FOLFIRINOX associated febrile neutropenia in patients with pancreatic cancer. Cancer Chemother. Pharm. 2020, 85, 651-659. [CrossRef]

12. Senter, P.D.; Beam, K.S.; Mixan, B.; Wahl, A.F. Identification and activities of human carboxylesterases for the activation of cpt-11, a clinically approved anticancer drug. Bioconjug. Chem. 2001, 12, 1074-1080. [CrossRef]

13. Xu, G.; Zhang, W.; Ma, M.K.; McLeod, H.L. Human carboxylesterase 2 is commonly expressed in tumor tissue and is correlated with activation of irinotecan. Clin. Cancer Res. 2002, 8, 2605-2611.

14. Dranitsaris, G.; Shah, A.; Spirovski, B.; Vincent, M. Severe diarrhea in patients with advanced-stage colorectal cancer receiving folfox or folfiri chemotherapy: The development of a risk prediction tool. Clin. Colorectal Cancer 2007, 6, 367-373. [CrossRef] [PubMed]

15. Shiozawa, T.; Tadokoro, J.; Fujiki, T.; Fujino, K.; Kakihata, K.; Masatani, S.; Morita, S.; Gemma, A.; Boku, N. Risk factors for severe adverse effects and treatment-related deaths in Japanese patients treated with irinotecan-based chemotherapy: A postmarketing survey. JPN J. Clin. Oncol. 2013, 43, 483-491. [CrossRef] [PubMed]

16. Innocenti, F.; Undevia, S.D.; Iyer, L.; Chen, P.X.; Das, S.; Kocherginsky, M.; Karrison, T.; Janisch, L.; Ramírez, J.; Rudin, C.M.; et al. Genetic variants in the udp-glucuronosyltransferase 1a1 gene predict the risk of severe neutropenia of irinotecan. J. Clin. Oncol. 2004, 22, 1382-1388. [CrossRef]

17. Iyer, L.; King, C.D.; Whitington, P.F.; Green, M.D.; Roy, S.K.; Tephly, T.R.; Coffman, B.L.; Ratain, M.J. Genetic predisposition to the metabolism of irinotecan (cpt-11). Role of uridine diphosphate glucuronosyltransferase isoform 1a1 in the glucuronidation of its active metabolite (sn-38) in human liver microsomes. J. Clin. Investig. 1998, 101, 847-854. [CrossRef]

18. Yang, Y.; Zhou, M.; Hu, M.; Cui, Y.; Zhong, Q.; Liang, L.; Huang, F. UGT1A1*6 and UGT1A1*28 polymorphisms are correlated with irinotecan-induced toxicity: A meta-analysis. Asia Pac. J. Clin. Oncol. 2018, 14, e479-e489. [CrossRef]

19. Hikino, K.; Ozeki, T.; Koido, M.; Terao, C.; Kamatani, Y.; Murakami, Y.; Kubo, M.; Mushiroda, T. Comparison of effects of UGT1A1*6 and UGT1A1*28 on irinotecan-induced adverse reactions in the Japanese population: Analysis of the biobank japan project. J. Hum. Genet. 2019, 64, 1195-1202. [CrossRef]

20. Ando, Y.; Saka, H.; Ando, M.; Sawa, T.; Muro, K.; Ueoka, H.; Yokoyama, A.; Saitoh, S.; Shimokata, K.; Hasegawa, Y. Polymorphisms of udp-glucuronosyltransferase gene and irinotecan toxicity: A pharmacogenetic analysis. Cancer Res. 2000, 60, 6921-6926. [PubMed]

21. Fujita, K.; Sparreboom, A. Pharmacogenetics of irinotecan disposition and toxicity: A review. Curr. Clin. Pharm. 2010, 5, 209-217. [CrossRef]

22. Nelson, R.S.; Seligson, N.D.; Bottiglieri, S.; Carballido, E.; Cueto, A.D.; Imanirad, I.; Levine, R.; Parker, A.S.; Swain, S.M.; Tillman, E.M. UGT1A1 guided cancer therapy: Review of the evidence and considerations for clinical implementation. Cancers 2021, 13, 1566. [CrossRef] [PubMed]

23. Gammal, R.S.; Court, M.H.; Haidar, C.E.; Iwuchukwu, O.F.; Gaur, A.H.; Alvarellos, M.; Guillemette, C.; Lennox, J.L.; Whirl-Carrillo, M.; Brummel, S.S.; et al. Clinical pharmacogenetics implementation consortium (cpic) guideline for UGT1A1 and atazanavir prescribing. Clin. Pharm. Ther. 2016, 99, 363-369. [CrossRef] [PubMed] 
24. Cho, I.R.; Kang, H.; Jo, J.H.; Lee, H.S.; Chung, M.J.; Park, J.Y.; Park, S.W.; Song, S.Y.; Chung, J.B.; An, C.; et al. Efficacy and treatment-related adverse events of gemcitabine plus nab-paclitaxel for treatment of metastatic pancreatic cancer "in a Korean" population: A single-center cohort study. Semin. Oncol. 2017, 44, 420-427. [CrossRef]

25. Eisenhauer, E.A.; Therasse, P.; Bogaerts, J.; Schwartz, L.H.; Sargent, D.; Ford, R.; Dancey, J.; Arbuck, S.; Gwyther, S.; Mooney, M.; et al. New response evaluation criteria in solid tumours: Revised recist guideline (version 1.1). Eur. J. Cancer 2009, 45, $228-247$. [CrossRef] [PubMed]

26. US Department of Health and Human Services; National Institutes of Health; National Cancer Institute. Common Terminology Criteria for Adverse Events (CTCAE) V5.0. Available online: https:/ / ctep.cancer.gov/protocoldevelopment/electronic_ applications/docs/CTCAE_v5_Quick_Reference_8.5x11.pdf (accessed on 31 August 2021).

27. Freifeld, A.G.; Bow, E.J.; Sepkowitz, K.A.; Boeckh, M.J.; Ito, J.I.; Mullen, C.A.; Raad, I.I.; Rolston, K.V.; Young, J.-A.H.; Wingard, J.R. Clinical practice guideline for the use of antimicrobial agents in neutropenic patients with cancer: 2010 update by the infectious diseases society of America. Clin. Infect. Dis. 2011, 52, e56-e93. [CrossRef]

28. Toffoli, G.; Cecchin, E.; Corona, G.; Russo, A.; Buonadonna, A.; D'Andrea, M.; Pasetto, L.M.; Pessa, S.; Errante, D.; De Pangher, V.; et al. The role of UGT1A1*28 polymorphism in the pharmacodynamics and pharmacokinetics of irinotecan in patients with metastatic colorectal cancer. J. Clin. Oncol. 2006, 24, 3061-3068. [CrossRef] [PubMed]

29. Toffoli, G.; Cecchin, E.; Gasparini, G.; D’Andrea, M.; Azzarello, G.; Basso, U.; Mini, E.; Pessa, S.; De Mattia, E.; Lo Re, G.; et al. Genotype-driven phase i study of irinotecan administered in combination with fluorouracil/leucovorin in patients with metastatic colorectal cancer. J. Clin. Oncol. 2010, 28, 866-871. [CrossRef]

30. Dias, M.M.; Pignon, J.P.; Karapetis, C.S.; Boige, V.; Glimelius, B.; Kweekel, D.M.; Lara, P.N.; Laurent-Puig, P.; Martinez-Balibrea, E.; Páez, D.; et al. The effect of the UGT1A1*28 allele on survival after irinotecan-based chemotherapy: A collaborative meta-analysis. Pharm. J. 2014, 14, 424-431. [CrossRef]

31. Innocenti, F.; Schilsky, R.L.; Ramírez, J.; Janisch, L.; Undevia, S.; House, L.K.; Das, S.; Wu, K.; Turcich, M.; Marsh, R.; et al. Dose-finding and pharmacokinetic study to optimize the dosing of irinotecan according to the UGT1A1 genotype of patients with cancer. J. Clin. Oncol. 2014, 32, 2328-2334. [CrossRef]

32. Fujii, H.; Yamada, Y.; Watanabe, D.; Matsuhashi, N.; Takahashi, T.; Yoshida, K.; Suzuki, A. Dose adjustment of irinotecan based on UGT1A1 polymorphisms in patients with colorectal cancer. Cancer Chemother. Pharm. 2019, 83, 123-129. [CrossRef]

33. Etienne-Grimaldi, M.C.; Boyer, J.C.; Thomas, F.; Quaranta, S.; Picard, N.; Loriot, M.A.; Narjoz, C.; Poncet, D.; Gagnieu, M.C.; Ged, C.; et al. UGT1A1 genotype and irinotecan therapy: General review and implementation in routine practice. Fundam. Clin. Pharm. 2015, 29, 219-237. [CrossRef]

34. Swen, J.J.; Nijenhuis, M.; de Boer, A.; Grandia, L.; Maitland-van der Zee, A.H.; Mulder, H.; Rongen, G.A.; van Schaik, R.H.; Schalekamp, T.; Touw, D.J.; et al. Pharmacogenetics: From bench to byte-An update of guidelines. Clin. Pharm. Ther. 2011, 89, 662-673. [CrossRef] [PubMed]

35. Berg, A.O.; Armstrong, K.; Botkin, J.; Calonge, N.; Haddow, J.; Hayes, M.; Kaye, C.; Phillips, K.A.; Piper, M.; Richards, C.S.; et al. Recommendations from the egapp working group: Can UGT1A1 genotyping reduce morbidity and mortality in patients with metastatic colorectal cancer treated with irinotecan? Genet. Med. 2009, 11, 15-20.

36. Shirasu, H.; Todaka, A.; Omae, K.; Fujii, H.; Mizuno, N.; Ozaka, M.; Ueno, H.; Kobayashi, S.; Uesugi, K.; Kobayashi, N.; et al. Impact of UGT1A1 genetic polymorphism on toxicity in unresectable pancreatic cancer patients undergoing FOLFIRINOX. Cancer Sci. 2019, 110, 707-716. [CrossRef]

37. Sharma, M.R.; Joshi, S.S.; Karrison, T.G.; Allen, K.; Suh, G.; Marsh, R.; Kozloff, M.F.; Polite, B.N.; Catenacci, D.V.T.; Kindler, H.L. A UGT1A1 genotype-guided dosing study of modified FOLFIRINOX in previously untreated patients with advanced gastrointestinal malignancies. Cancer 2019, 125, 1629-1636. [CrossRef] [PubMed]

38. Joshi, S.S.; Catenacci, D.V.T.; Karrison, T.G.; Peterson, J.D.; Zalupski, M.M.; Sehdev, A.; Wade, J.; Sadiq, A.; Picozzi, V.J.; Amico, A.; et al. Clinical assessment of 5-fluorouracil/leucovorin, nab-paclitaxel, and irinotecan (FOLFIRABRAX) in untreated patients with gastrointestinal cancer using UGT1A1 genotype-guided dosing. Clin. Cancer Res. 2020, 26, 18-24. [CrossRef]

39. de Man, F.M.; Goey, A.K.L.; van Schaik, R.H.N.; Mathijssen, R.H.J.; Bins, S. Individualization of irinotecan treatment: A review of pharmacokinetics, pharmacodynamics, and pharmacogenetics. Clin. Pharm. 2018, 57, 1229-1254. [CrossRef] [PubMed]

40. Cheng, L.; Li, M.; Hu, J.; Ren, W.; Xie, L.; Sun, Z.P.; Liu, B.R.; Xu, G.X.; Dong, X.L.; Qian, X.P. UGT1A1*6 polymorphisms are correlated with irinotecan-induced toxicity: A system review and meta-analysis in Asians. Cancer Chemother. Pharm. 2014, 73, 551-560. [CrossRef] [PubMed]

41. Kim, J.J.; Oh, J.; Kim, Y.; Lee, K.A. Genetic spectrum of UGT1A1 in Korean patients with unconjugated hyperbilirubinemia. Ann. Lab. Med. 2020, 40, 281-283. [CrossRef]

42. Minami, H.; Sai, K.; Saeki, M.; Saito, Y.; Ozawa, S.; Suzuki, K.; Kaniwa, N.; Sawada, J.; Hamaguchi, T.; Yamamoto, N.; et al. Irinotecan pharmacokinetics/pharmacodynamics and ugt1a genetic polymorphisms in Japanese: Roles of UGT1A1*6 and *28. Pharm. Genom. 2007, 17, 497-504. [CrossRef]

43. Hu, Z.Y.; Yu, Q.; Pei, Q.; Guo, C. Dose-dependent association between UGT1A1*28 genotype and irinotecan-induced neutropenia: Low doses also increase risk. Clin. Cancer Res. 2010, 16, 3832-3842. [CrossRef] [PubMed]

44. Hoskins, J.M.; Goldberg, R.M.; Qu, P.; Ibrahim, J.G.; McLeod, H.L. UGT1A1*28 genotype and irinotecan-induced neutropenia: Dose matters. J. Natl. Cancer Inst. 2007, 99, 1290-1295. [CrossRef] [PubMed] 
45. Umemoto, K.; Takahashi, H.; Morizane, C.; Yamada, I.; Shimizu, S.; Shioji, K.; Yoshida, Y.; Motoya, M.; Mizuno, N.; Kojima, Y.; et al. Folfirinox in advanced pancreatic cancer patients with the double-variant type of UGT1A1*28 and *6 polymorphism: A multicenter, retrospective study. Cancer Chemother. Pharm. 2021, 87, 397-404. [CrossRef] [PubMed]

46. Innocenti, F.; Kroetz, D.L.; Schuetz, E.; Dolan, M.E.; Ramírez, J.; Relling, M.; Chen, P.; Das, S.; Rosner, G.L.; Ratain, M.J. Comprehensive pharmacogenetic analysis of irinotecan neutropenia and pharmacokinetics. J. Clin. Oncol. 2009, 27, 2604-2614. [CrossRef]

47. Crawford, J.; Glaspy, J.A.; Stoller, R.G.; Tomita, D.K.; Vincent, M.E.; McGuire, B.W.; Ozer, H. Final results of a placebo-controlled study of filgrastim in small-cell lung cancer: Exploration of risk factors for febrile neutropenia. Support Cancer Ther. 2005, 3, 36-46. [CrossRef] [PubMed]

48. Lyman, G.H.; Abella, E.; Pettengell, R. Risk factors for febrile neutropenia among patients with cancer receiving chemotherapy: A systematic review. Crit. Rev Oncol. Hematol. 2014, 90, 190-199. [CrossRef]

49. Jung, J.H.; Shin, D.W.; Kim, J.; Lee, J.C.; Hwang, J.H. Primary granulocyte colony-stimulating factor prophylaxis in metastatic pancreatic cancer patients treated with FOLFIRINOX as the first-line treatment. Cancers 2020, 12, 3137. [CrossRef] [PubMed]

50. Liu, C.Y.; Chen, P.M.; Chiou, T.J.; Liu, J.H.; Lin, J.K.; Lin, T.C.; Chen, W.S.; Jiang, J.K.; Wang, H.S.; Wang, W.S. UGT1A1*28 polymorphism predicts irinotecan-induced severe toxicities without affecting treatment outcome and survival in patients with metastatic colorectal carcinoma. Cancer 2008, 112, 1932-1940. [CrossRef]

51. Loh, M.; Chua, D.; Yao, Y.; Soo, R.A.; Garrett, K.; Zeps, N.; Platell, C.; Minamoto, T.; Kawakami, K.; Iacopetta, B.; et al. Can population differences in chemotherapy outcomes be inferred from differences in pharmacogenetic frequencies? Pharm. J. 2013, 13, 423-429. [CrossRef]

52. Rosmarin, D.; Palles, C.; Church, D.; Domingo, E.; Jones, A.; Johnstone, E.; Wang, H.; Love, S.; Julier, P.; Scudder, C.; et al. Genetic markers of toxicity from capecitabine and other fluorouracil-based regimens: Investigation in the quasar2 study, systematic review, and meta-analysis. J. Clin. Oncol. 2014, 32, 1031-1039. [CrossRef] [PubMed]

53. Yap, Y.S.; Kwok, L.L.; Syn, N.; Chay, W.Y.; Chia, J.W.K.; Tham, C.K.; Wong, N.S.; Lo, S.K.; Dent, R.A.; Tan, S.; et al. Predictors of hand-foot syndrome and pyridoxine for prevention of capecitabine-induced hand-foot syndrome: A randomized clinical trial. JAMA Oncol. 2017, 3, 1538-1545. [CrossRef] [PubMed] 